Résumés des conférences et travaux

\title{
Histoire et philologie tibétaines
}

\section{Charles Ramble}

\section{(2) OpenEdition Journals}

Édition électronique

URL : https://journals.openedition.org/ashp/2737

DOI : $10.4000 /$ ashp. 2737

ISSN : 1969-6310

Éditeur

Publications de l'École Pratique des Hautes Études

\section{Édition imprimée}

Date de publication : 1 septembre 2018

Pagination : 382-387

ISSN : 0766-0677

\section{Référence électronique}

Charles Ramble, "Histoire et philologie tibétaines », Annuaire de l'École pratique des hautes études (EPHE), Section des sciences historiques et philologiques [En ligne], 149 | 2018, mis en ligne le 11 juillet 2018, consulté le 03 août 2021. URL : http://journals.openedition.org/ashp/2737 ; DOI : https:// doi.org/10.4000/ashp. 2737 


\title{
HISTOIRE ET PHILOLOGIE TIBÉTAINES
}

\author{
Directeur d'études : M. Charles Ramble
}

Programme de l'année 2016-2017 : I. Sources tibétaines pour l'étude de l'origine et la soumission de vampires (sri). - II. Kun-bzang chos-gling : histoire et administration d'un couvent tibétain dans l'Himalaya, 1670-1940.

\section{Sources tibétaines pour l'étude de l'origine et la soumission de vampires (sri)}

Cette année a clôturé une période de trois ans consacrée à l'étude des textes concernant les vampires dans la croyance tibétaine. Après nous être concentré sur la mythologie et les techniques de soumission de ces démons, nous nous sommes intéressé cette année au cadre plus large dans lequel ces rituels sont inscrits. Un manuscrit en particulier, que nous avons photographié en 2008 dans le district de Mustang au Népal, a formé la base de l'enseignement. Il s'agit d'une œuvre de 19 folios, écrite en script « sans tête » (dbu med), appartenant à Lama Tshultrim du village de Lubrak. L'ouvrage s'intitule Bon skyongs rgyun tshogs nar ma'i dbu phyogs, «L'offrande régulière de nourriture consacrée aux protecteurs du Bon ». Le rituel basé sur ce texte est connu sous le nom de gtor bskul, « Incitation au moyen de gtor ma [gâteaux sacrificiels] », et se retrouve dans plusieurs performances exorcistes ou apotropaïques. Comme le suggère le titre, le but est d'inciter ([b]skul) les divinités protectrices représentées par les gtor $m a$ à repousser les puissances hostiles et, en particulier, à attaquer le vampire. Le contenu du texte a été mis en rapport avec une performance rituelle réelle que nous avons filmée en 2013. Une quarantaine de divinités, ou catégories de divinités, sont invoquées à tour de rôle. Chacune est décrite en détail, précisant sa couleur, son comportement, ses attributs et, le cas échéant, son entourage. Le texte rappelle à plusieurs reprises aux divinités leur serment originel de servir le Bon et leur offre des gtor ma joliment décorés en échange de la protection de la doctrine et du prêtre. L'invocation implique une distinction significative entre ces effigies : alors que certaines gtor ma sont simplement des offrandes, d'autres sont les supports des protecteurs. Précédant l'invocation du dernier ensemble de la série de ces protecteurs, les Filets Rouges et Noirs (Dra ba dmar nag), sont listés les rituels subsidiaires pour les cinq divinités transcendantes : Kun bzang a gsal; dBal gsas; sTag lha; Khro rgyal sPyi 'dul, et Srid pa'i rgyal mo - cette dernière a déjà été invoquée dans la liste des protecteurs en vertu de sa double appartenance aux deux catégories. Certaines des divinités invoquées, en effet, sont des dieux transcendants, les yi dam, mais les véritables bénéficiaires des offrandes sont les protecteurs mondains qui constituent leur entourage. Chacun de ces cinq rituels est présenté comme un dmar lam, un type d'offrande de gtor $m a$, dans lequel les offrandes sont particulièrement somptueuses. Bien que dmar implique que ce rite comporte des offrandes «rouges », le premier qui invoque la divinité paisible Kun bzang a gsal, ne spécifie pas d'offrandes rouges (viande, sang...). Dans certains cas, ce n'est pas la divinité centrale, le yi dam, mais 
seulement les protecteurs dans son entourage qui sont honorés. Précisons enfin que même si toutes les divinités répertoriées ne sont pas représentées sous forme de gtor ma sur l'autel pendant le rituel, le texte est à chaque fois récité dans son intégralité.

Au cours du deuxième semestre, M. Alexander Smith, chercheur post-doctoral à l'université d'Erlangen, a donné un cours hebdomadaire sur le thème de la divination dans la religion Bon. À cette fin, il a examiné des traductions choisies parmi un certain nombre de manuscrits de cléromancie, principalement composés entre les $\mathrm{XIV}^{\mathrm{e}}$ et $\mathrm{XX}^{\mathrm{e}}$ siècles. Les leçons ont porté sur les origines mythiques de la divination telles qu'elles sont représentées dans la tradition ecclésiastique du Bon, ainsi que sur les diverses divinités et dispositifs symboliques couramment rencontrés dans les invocations divinatoires. Dans la première moitié du semestre, il s'est principalement concentré sur des extraits du $m D o$ 'dri med gzi brjid, ouvrage hagiographique du $\mathrm{XIV}^{\mathrm{e}}$ siècle qui contient plusieurs récits mythiques non étudiés concernant la création de la divination par les dieux et sa diffusion dans le monde des hommes. Ces récits ont ensuite été comparés à des manuels de divination plus récents provenant du Tibet central et de l'Ouest du Népal. Dans la seconde moitié du semestre, M. Smith a lu des extraits de deux manuels sur une forme rare de divination à l'aide de cordelettes appelée $j u$ thig. Ces textes offrent une riche variété de matériaux mythologiques, incluant les thèmes de l'élevage et des pratiques horticoles dans les sociétés tibétaines qui ont suscité de vives discussions.

\section{Kun-bzang chos-gling : histoire et administration d'un couvent tibétain dans l'Himalaya, 1670-1940}

Cette année, nous avons exploré l'histoire d'un couvent bouddhiste tibétain, Künzang Chöling (Kun bzang chos gling) sur la base de manuscrits que nous avons photographiés au Népal en 1993 et en 2016. Ce couvent, à présent abandonné, se trouve sur le territoire de Tshug, un grand village dans la région de Shöyul (Shod yul) dans le Mustang méridional. David Snellgrove, qui l'a visité en 1956, le décrit dans le passage suivant :

The grandeur of this temple, still impressive in spite of its present dilapidated condition, its remoteness, to which the swift-flowing river adds yet another barrier, reminded me of that still more ancient monastery of Tabo in Spiti. They are both places which haunt the imagination and to which one feels an urge to return. It is known locally just as Gonpa K'ang, "Promontory Monastery", but its proper name is Kun-bzang chos-gling, "AllGood Island of the Doctrine". Once the centre of a large community, it is now watched over by a single monk, a Tibetan from Drepung, who finds it a congenial place for his meditation. (Snellgrove $1961:$ 173)

Comme le remarque Snellgrove, le site est mieux connu sous le nom de Gönpa Gang (dGon pa sgang), «le monastère du promontoire ». Bien que cette traduction semble assez naturelle, le nom tibétain suggère plutôt d'inverser les termes, ce qui nous donne «le promontoire du monastère »- ou, plus précisément, « la colline du couvent ", sgang étant le terme habituel dans le dialecte du Mustang pour désigner une colline : et en effet, nous savons maintenant que lorsque la construction de Künzang Chöling a commencé en 1684, le nom Gönpa Gang était déjà utilisé pour 
désigner un ou plusieurs établissements religieux qui se trouvaient sur le site - probablement dans des grottes dans la falaise qui surplombe le site à l'ouest. Gönpa Gang était précisément la colline d'un ou plusieurs établissements religieux. Après cette période, les deux noms semblent avoir été utilisés de manière interchangeable. Dans les documents examinés, comme dans l'usage quotidien au Mustang aujourd'hui, le nom Gönpa Gang a complètement remplacé Künzang Chöling.

\section{Des sources récemment découvertes}

Jusqu'à très récemment, on ne savait presque rien de l'histoire de ce couvent. En 1993, nous avons photographié les archives du village de Tshug dans le cadre du projet «Nepal-German Project on High Mountain Archaeology », un projet financé par le Conseil allemand de la recherche (DFG) sous la direction de Dieter Schuh. Une classification préliminaire de cette collection a révélé que si la majorité des documents traitaient des affaires du village, une vingtaine concernaient directement le couvent. Au cours de cette visite, nous avons également pu photographier les archives du couvent lui-même, une vingtaine d'autres objets conservés dans une maison privée. Il y a donc deux collections de documents qui concernent le couvent : les archives de Kunzang Choling lui-même et certains documents des archives de la communauté Tshug.

Cependant ces documents ne nous disent rien sur les origines du couvent : quand, et par qui, il a été fondé. C'est seulement en 2016, lors d'une visite à Tshug, que nous avons eu l'occasion de photographier un manuscrit de la biographie du fondateur. Cet ouvrage s'intitule Rang gi rtog pa brjod pa kun bzang dgongs ba rang shar zhes par ba bzhugs +ho, et comprend 165 folios écrits dans l'écriture dite « sans tête » dbu med.

D'après ce nouveau document, le fondateur de Künzang Chöling, Künzang Longyang, était le disciple et le successeur d'Orgyan Palzang (O rgyan dpal bzang). Un certain nombre de ses activités ont été décrites par F.-K. Ehrhard (2013a-d), qui tire la plupart de ses informations d'une biographie de ce personnage. Le titre qu'Ehrhard donne pour cette œuvre est exactement le même (y compris l'orthographe erronée de chun comme mchun) que le titre de la deuxième partie de la biographie que nous avons photographiée et qui contient les chansons de l'auteur et les enseignements qu'il a reçus, mais le texte n'est pas le même. Il est clair, à partir des passages cités par Ehrhard, que ce travail est sensiblement le même que la première partie du manuscrit de Tshug - l'autobiographie proprement dite. Cependant, il semble que ce soit une version abrégée de notre texte, car il omet certains événements dramatiques qu'Ehrhard n'aurait pas manqué de mentionnerés : Tshene Gyalpo (Tshe gnas rgyal po), duc de Baragaon et principal mécène de Künzang Longyang a été tué lors de la destruction de son château, Dzong Rabgyaltse, dans le tremblement de terre de 1680. Encore plus étonnant, la version de l'autobiographie utilisée par Ehrhard omet le fait que Künzang Longyang était le fondateur de Künzang Chöling.

Les ancêtres de l'auteur étaient originaires de Dzar (Jharkot en népalais) dans la vallée de Muktinath, un clan aristocratique appelé Dzar Pöngyü (Dzar dpon brgyud) - littéralement « ligne noble de Dzar », mais en réalité un nom propre. Une branche de la famille s'est installé à Dolpo, où Künzang Longyang est né le mercredi 5 juin 1644. En tant que fils aîné, il était censé devenir chef de famille, et ses inclinations spirituelles causèrent une anxiété considérable à ses parents. 
L'une de ses premières rencontres les plus significatives a eu lieu à l'âge de quinze ans, avec Chöje Karma Zangpo, un disciple de Marpa le traducteur. Il devint plus tard un disciple d'Orgyen Palzang (O rgyan dpal bzang), lui-même disciple du grand Düdul Dorje (bDud 'dul rdo rje) au Tibet oriental. Orgyen Palzang lui a donné le nom de Künzang Longyang. Le jeune moine nouvellement ordonné est alors allé à la rencontre de son maître qui faisait une retraite solitaire dans son Sompo (Thini) natal pour finalement découvrir que la construction d'un temple sur la crête appelée la Colline de la Lumière Claire, Ösal Gang ('Od gsal sgang), était déjà en cours. Künzang Longyang adopta Ösal Gang comme nouvelle demeure spirituelle et, après la mort de son maître, lui succéda en tant que lama supérieur de son temple, Kutshab Ternga, dans sa $34^{\mathrm{e}}$ année.

Künzang Longyang n'a pas tardé à recevoir des invitations pour officier dans des cérémonies dans les communautés voisines. L'une d'entre elles était une invitation à une cérémonie à Tshug par une moniale nommée Sherab (Shes rab) de Gonpa Gang. C'est la première indication dans l'autobiographie que Gonpa Gang était déjà le site d'au moins un établissement religieux avant que Künzang Longyang construise son couvent une décennie plus tard. Une autre invitation importante qu'il reçut à cette époque était de Tshene Gyalpo, le seigneur de Baragaon qui résidait dans le palais de Rabgyaltse (Rab rgyal rtse), à Dzong, situé en haut de la vallée de Muktinath.

En 1683, alors qu'il était dans sa quarantième année, il reçut la nouvelle que son frère cadet était décédé. La perte de son frère semble avoir été l'événement qui l'a poussé à se lancer dans un projet ambitieux qu'il envisageait depuis quelque temps : la construction du temple qui devait devenir Künzang Chöling. L'ensemble du projet, du début à la fin, durerait douze ans.

Lors d'une visite ultérieure à son domicile à Dolpo, il a constaté que sa mère avait beaucoup vieilli. Elle était infirme et pratiquement aveugle, et en effet elle est morte peu après. Il a ensuite entrepris un projet qu'il décrit comme un nang rten; Puisque ce terme désigne généralement une image commémorative pour quelqu'un qui est décédé, il est probable que la prochaine phase de construction ait été inspirée par la perte de sa mère. Après une période de collecte de fonds au Dolpo, la construction du nang rten lui-même - une grande image du futur bouddha Maitreya - a pu commencer.

Mustang à cette époque était vassal du royaume de Jumla. Vers la fin de la biographie, est mentionnée une rencontre avec le ministre du roi, qui a accompagné Künzang Longyang lors d'une visite à son nouveau temple.

Il s'est étonné des décorations à l'extérieur comme à l'extérieur du bâtiment. « Lama ", dit-il, « ce temple que vous avez construit ici est le joyau de la couronne du roi de Jumla. Il rehausse tout particulièrement le prestige du roi. Quelle belle chose vous avez faite ici ! », et il m'a grandement félicité. Il m'a demandé des charmes pour dissiper certaines conditions involontaires et repousser les maux, et je lui ai donc donné des talismans, etc. Il était très heureux. Je pensais que, même si les hindous ne sont pas concernés par l'accumulation de vertus ni par le renoncement aux péchés, quelqu'un comme lui, croyant en ces objets sacrés, trouverait le bonheur dans le futur. (f. 85 r)

Künzang Longyang est décédé peu de temps après, « sans douleur ni souffrance ». 


\section{Les archives de Künzang Chöling}

Les activités cérémonielles de Künzang Chöling sont consignées dans deux documents. La plupart des membres de la communauté auraient résidé dans leurs maisons familiales pendant la plus grande partie de l'année et ne se seraient rassemblés au couvent qu'à ces occasions. Les cérémonies énumérées sont liées aux noms des individus des communautés voisines. Certains étaient probablement les parrains de ces événements, alors que ceux associés aux prières étaient les défunts bénéficiaires.

La principale source de financement des activités cérémonielles du couvent était un système de dotation connu sous le nom de sbyor 'jag. Les gens des villages environnants - à commencer par Tshug lui-même - faisaient des dons en espèces au couvent. Cet argent était ensuite prêté et l'emprunteur versait au couvent un intérêt annuel de $1 \%$ sous forme d'orge. Le ratio grain / espèce nous donne une idée de la valeur de la roupie. Pendant la majeure partie de la période considérée, la valeur de la roupie était 1500 fois supérieure à ce qu'elle est actuellement; en 1774, date du premier document, il aurait été 9000 fois plus grand.

Des dossiers annuels sur les personnes à qui les prêts ont été consentis ont été tenus et ces notes ont été compilées périodiquement dans des registres de prêts couvrant plusieurs décennies. Dans plusieurs cas, le nom de l'emprunteur est suivi du nom d'un garant financièrement responsable en cas de défaut de paiement des intérêts. Les nonnes elles-mêmes pouvaient prétendre à des prêts. Comme les emprunteurs non professionnels, certaines étaient considérées comme financièrement plus fiables que d'autres et ne nécessitaient pas de caution. Le retour sur les investissements du couvent a été complété par le rendement des champs qui avaient été donnés par les dévots.

Il n'est pas rare que les emprunteurs meurent avant d'avoir payé leurs dettes et, au moins une fois, le couvent rédigea un document concernant ses créanciers mais avec les noms de leurs héritiers. Malgré le soutien de ses créanciers et d'autres mécènes, Künzang Chöling semble avoir traversé des périodes de difficultés financières. À une occasion, le lama responsable, nommé Nyildabum (Nyi zla 'bum), congédia les religieuses de Tshug pour une période de trois ans, mais les religieuses réussirent à persuader la communauté laïque de fournir une somme importante qui permit au couvent de survivre, toutefois pas très longtemps.

Le couvent était gravement affaibli en 1903 lorsque les religieuses de Te, un des deux principaux villages de la paroisse, se retirèrent de Künzang Chöling et établirent une communauté séparée sous la tutelle d'un lama local. Künzang Chöling ne se remit jamais complètement de cette séparation.

\section{Les règles de Gönpa Gang}

L'ensemble original des règles du couvent n'a pas survécu, mais deux autres documents de ce type ont été rédigés respectivement en 1890 et 1942 . Ces règles couvrent un large éventail de questions comme la spécification des dates auxquelles les religieuses doivent être présentes pour les cérémonies au couvent, ce qui souligne le besoin de ponctualité, et la nécessité d'une présence au couvent pendant la durée de chaque cérémonie. Ces règles stipulaient aussi les mesures à prendre en cas de grossesse, selon que la religieuse épouse le père de l'enfant et devienne laïque (processus 
appelé mar log, « retourner vers le bas »), ou qu'elle reste célibataire et retourne au couvent en tant que religieuse dans un delai de trois ans (yar log, « retourner vers le haut »). La troisième possibilité consistait à rester célibataire sans revenir au couvent, étant « ni nonne ni laïque » (jo min skya min), une mesure particulièrement désapprouvée qui entraînait la plus lourde pénalité financière. Le deuxième document interdit également l'enlèvement des nonnes pour les épouser.

\section{Ouvrages cités}

Ehrhard, Franz-Karl. 2013a. « Tibetan sources on Muktināth: individual reports and normative guides », dans Buddhism in Tibet and the Himalayas: Text and Traditions, Kathmandu, Vajra Publications, p. 201-217.

— 2013b. «Concepts of religious space in Southern Mustang: the foundation of the monastery sKu-tshab gter-lnga ", dans Buddhism in Tibet and the Himalayas: Texts and Traditions, Kathmandu, Vajra Publications, p. 218-228.

- 2013c. «Religious geography and literary traditions: the foundation of the monastery Bragdkar bsam-gling ", dans Buddhism in Tibet and the Himalayas: Texts and Traditions, Kathmandu, Vajra Publications, p. 229-238.

- 2013d. "Pilgrims in search of sacred lands ", dans Buddhism in Tibet and the Himalayas: Texts and Traditions, Kathmandu, Vajra Publications, p. 239-256.

Snellgrove, D. L. 1989 [1961]. Himalayan Pilgrimage: A Study of Tibetan Religion by a Traveller through Western Nepa, Boston, Shambhala. 